\title{
Assessing the Role and Optimal Duration of Hormonal Treatment in Association with Salvage Radiation Therapy After Radical Prostatectomy: Results from a Multi-Institutional Study
}

Nicola Fossati

Corresponding author.

Division of Oncology/Unit of Urology, IRCCS Ospedale San Raffaele, Vita-Salute San Raffaele University, Via

Olgettina 60, Milan 20131, Italy.

Tel. +3902 26437286;

Fax: +3902 26437298.

E-mail address: fossati.nicola@hsr.it

Daniele Robesti

Division of Oncology/Unit of Urology, Urological Research Institute, IRCCS Ospedale San Raffaele, Milan, Italy

R. Jeffrey Karnes

Department of Urology, Mayo Clinic, Rochester, MN, USA

Matteo Soligo

Department of Urology, Mayo Clinic, Rochester, MN, USA

Stephen A. Boorjian

Department of Urology, Mayo Clinic, Rochester, MN, USA

Alberto Bossi

Department of Radiation Oncology, Gustave Roussy Institute, Villejuif, France

Gabriele

Coraggio

Department of Radiation Oncology, Gustave Roussy Institute, Villejuif, France

Nadia Di Muzio

Department of Radiotherapy, IRCCS Ospedale San Raffaele, Milan, Italy

Cesare Cozzarini

Department of Radiotherapy, IRCCS Ospedale San Raffaele, Milan, Italy

Barbara Noris Chiorda

Department of Radiotherapy, IRCCS Ospedale San Raffaele, Milan, Italy

Giorgio Gandaglia

Division of Oncology/Unit of Urology, Urological Research Institute, IRCCS Ospedale San Raffaele, Milan, Italy

Simone Scarcella

Division of Oncology/Unit of Urology, Urological Research Institute, IRCCS Ospedale San Raffaele, Milan, Italy 
Detlef Bartkowiak

Department of Radiation Oncology, University Hospital Ulm, Ulm, Germany

Dirk Böhmer

Department of Radiation Oncology, Charité University Medicine, Berlin, Germany

Shahrokh Shariat

Department of Urology, Medical University of Vienna, Vienna, Austria

Gregor Goldner

Department of Radiation Oncology, Medical University of Vienna, Vienna, Austria

Antonino Battaglia

Department of Urology, University Hospitals Leuven, Leuven, Belgium

Steven Joniau

Department of Urology, University Hospitals Leuven, Leuven, Belgium

Karin Haustermans

Department of Radiation Oncology, University Hospitals Leuven, Leuven, Belgium

Gert De Meerleer

Department of Radiation Oncology, University Hospitals Leuven, Leuven, Belgium

Valérie Fonteyne

Department of Radiotherapy, Ghent University Hospital, Ghent, Belgium

Piet Ost

Department of Radiotherapy, Ghent University Hospital, Ghent, Belgium

Hein Van Poppel

Department of Urology, University Hospitals Leuven, Leuven, Belgium

Francesco Montorsi

Division of Oncology/Unit of Urology, Urological Research Institute, IRCCS Ospedale San Raffaele, Milan, Italy

Thomas Wiegel

Department of Radiation Oncology, University Hospital UIm, Ulm, Germany

Alberto Briganti

Division of Oncology/Unit of Urology, Urological Research Institute, IRCCS Ospedale San Raffaele, Milan, Italy 


\section{$\underline{\text { ABSTRACT }}$}

\section{BACKGROUND:}

The optimal duration of hormonal therapy $(\mathrm{HT})$ when associated with postprostatectomy radiation therapy (RT) remains controversial.

\section{OBJECTIVE:}

To test the impact of HT duration among patients treated with postprostatectomy RT, stratified by clinical and pathologic characteristics.

\section{DESIGN, SETTING, AND PARTICIPANTS:}

The study included 1264 patients who received salvage RT (SRT) to the prostatic and seminal vesicle bed at eight referral centers after radical prostatectomy (RP). Patients received SRT for either rising prostate-specific antigen (PSA) or PSA persistence after RP, defined as PSA $\geq 0.1 \mathrm{ng} / \mathrm{ml}$ at $1 \mathrm{mo}$ after surgery. Administration of concomitant HT was at the discretion of the treating physician.

\section{OUTCOME MEASUREMENTS AND STATISTICAL ANALYSIS:}

The outcome of interest was clinical recurrence (CR) after SRT, as identified by imaging. Multivariable Cox regression analysis was used to test the association between CR and HT duration. We applied an interaction test between HT duration and baseline risk factors to assess the hypothesis that CR-free survival differed by HT duration according to patient profile. Three risk factors were prespecified for evaluation: $\mathrm{pT}$ stage $\geq \mathrm{pT} 3 \mathrm{~b}$, pathologic Gleason $\geq 8$, and PSA level at SRT $>0.5 \mathrm{ng} / \mathrm{ml}$. The relationship between $\mathrm{HT}$ duration and $\mathrm{CR}$-free survival rate at $8 \mathrm{yr}$ was graphically explored according to the number of risk factors ( 0 vs 1 vs $\geq 2$ ).

RESULTS AND LIMITATIONS:

Overall, 1125 men $(89 \%)$ received SRT for rising PSA and 139 (11\%) were treated for PSA persistence. Concomitant HT was administered to 363 patients (29\%), with a median HT duration of 9 mo. At median follow-up of $93 \mathrm{mo}$ after surgery, 182 patients developed CR. The 8 -yr CR-free survival was $92 \%$. On multivariable analysis, HT duration was inversely associated with the risk of CR (hazard ratio 0.95; $\mathrm{p}=0.022$ ). A total of $531(42 \%)$ patients had none of the prespecified risk factors, while $507(40 \%)$ had one and $226(18 \%)$ had two or more risk factors. The association between HT duration and CR was significantly different by risk factors ( 0 vs $1, p=0.001 ; 0$ vs $\geq 2, p<0.0001$ ). We observed a significant effect of HT duration for patients with two or more risk factors, for whom HT administration was beneficial when given for up to $36 \mathrm{mo}$. This effect was attenuated among patients with one risk factor, with concomitant HT slightly beneficial when administered for a shorter time $(<12 \mathrm{mo})$. Conversely, for patients with no risk factors, the risk of CR remained low and constant regardless of HT duration.

\section{CONCLUSIONS:}

The oncologic benefit of HT duration among men receiving SRT for increasing PSA after RP depends on their clinical and pathologic characteristics. Our data suggested a significant effect of long-term HT for patients with two or more adverse features. Conversely, short-term HT was sufficient for patients with a single risk factor, whereas patients without any risk factors did not show a significant benefit from concomitant HT.

\section{PATIENT SUMMARY:}

We tested the impact of hormonal therapy $(\mathrm{HT})$ duration during radiation therapy after radical prostatectomy. We identified three risk factors and observed a different impact of HT duration by clinical and pathologic characteristics. Patients with more adverse features benefit from long-term concomitant HT. On the contrary, for patients with a single risk factor, short-term HT may be reasonable. Patients without any risk factors did not show a significant benefit from concomitant HT. 


\section{KEYWORDS}

Prostatic neoplasms

Neoplasm recurrence

Biochemical tumor markers

Radiotherapy

Salvage therapy

Hormonal therapy

\section{Introduction}

The management of prostate-specific antigen (PSA) recurrence after radical prostatectomy (RP) is a clinically significant issue for urologists and radiation oncologists, as approximately $30 \%$ of patients experience an increase in PSA at long-term follow-up after surgical treatment [1], [2], [3], [4], [5].

The addition of concomitant hormonal therapy $(\mathrm{HT})$ to postoperative radiation therapy (RT) is still an open question and remains controversial [6], [7]. Recent randomized clinical trials (RCTs) have shown that the addition of HT to postoperative RT improves oncologic outcomes. In particular, the RTOG 9601 [8] trial showed better overall survival when $2 \mathrm{yr}$ of bicalutamide was combined with salvage RT (SRT); the greatest benefit was observed in subgroups of patients with more aggressive cancer. Moreover, in the GETUG-AFU 16 trial [9], patients assigned to RT plus goserelin had lower biochemical progression and clinical progression rates at $5 \mathrm{yr}$ compared to patients treated with RT alone.

However, use of androgen deprivation therapy (ADT) is associated with a decrease in quality of life due to cardiovascular morbidity [10], [11], metabolic effects [12], nonmetastatic bone fractures [13], [14], sexual dysfunction [15], hot flushes [16], fatigue, and neurologic side effects [17]. The rate and the extent of these side effects depend on the HT duration [18], [19].

Therefore, the potential benefit of ADT should be balanced against the associated harms. Moreover, HT duration may have a differential effect according to the clinical and pathologic characteristics of patients. Thus, we hypothesized that the impact of HT duration during RT varies according to patient and tumor characteristics at final pathology. To test this hypothesis we evaluated a large multiinstitutional series of patients treated with post-RP RT.

\section{Patients and methods}

\subsection{Patient population}

We identified 1392 patients treated with SRT at eight tertiary referral centers between 1996 and 2012. All patients had histologically confirmed pT2-4 pNO-Nx adenocarcinoma of the prostate. Salvage RT was administered for either rising PSA during follow-up after RP or PSA persistence immediately after RP. Specifically, rising PSA after surgery was defined as undetectable PSA with a subsequent PSA increase within two or more measurements [20], whereas PSA persistence was defined as a serum concentration $\geq 0.1 \mathrm{ng} / \mathrm{ml}$ at $1 \mathrm{mo}$ after RP [21].

Patients with missing information for pathologic Gleason score $(n=8)$, PSA level at the time of RT $(n=30)$, or duration of concomitant HT $(n=90)$ were excluded. These selection criteria yielded 1264 evaluable individuals with complete clinical, pathologic, and follow-up data.

\subsection{RT technique}

$\mathrm{RT}$ consisted of local radiation to the prostate and seminal vesicle bed. All patients were treated with high-energy photon beams (10-25 MV) using conventional fractionation (1.8-2 Gy per fraction), with a median dose of $66 \mathrm{~Gy}$ (interquartile range [IQR] 63-66). Conventional nonconformal treatment was delivered and rectangular or minimally blocked beams were used. Alternatively, a three-dimensional conformal approach was used. The clinical target volume (CTV) was delineated on computed 
tomography (CT) images and included the prostatic fossa and periprostatic tissue. Clinical findings, preoperative $\mathrm{CT}$ images, and surgical clips guided the clinicians for CTV definition. The planned target volume was defined as CTV plus a margin of $0.8-1.0 \mathrm{~cm}$ to account for organ motion and setup error. Whole-pelvis RT was administered to 430 patients (24\%). The indication for irradiation of the pelvic lymph node area was based on the clinical judgment of each treating physician, according to individual patient and cancer characteristics.

\subsection{HT concomitant to SRT}

The indication for concomitant $\mathrm{HT}$, the type of drug administered, and the treatment duration were left at the discretion of the treating physician on the basis of individual patient characteristics.

\subsection{Variable definitions}

Clinical and pathologic data consisted of patient age at RP, preoperative PSA level, pathologic stage (pT2 vs pT3a vs $\geq$ pT3b), pathologic Gleason score ( $\leq 6$ vs 7 vs $\geq 8$ ), surgical margin status (negative vs positive), lymph node status ( $\mathrm{pNO}$ vs pNx), undetectable postoperative PSA (no vs yes), PSA level at RT, PSA doubling time (for patients treated for rising PSA), RT field (prostatic fossa vs whole pelvis), RT dose, concomitant HT administration (no vs yes), and duration of concomitant HT (which was set to 0 for patients who did not receive concomitant HT).

\subsection{Outcome definitions}

The study outcome was clinical recurrence (CR) after RT, which included local recurrence, pelvic and retroperitoneal lymph node metastasis, skeletal metastasis, and visceral metastasis. CR was identified by radiologic imaging and confirmed via biopsy at the discretion of the treating clinician. Follow-up time was defined as the time elapsed between RT and the onset of CR or last follow-up.

\subsection{Statistical analysis}

Our statistical analysis consisted of four main steps. First, multivariable Cox regression analysis tested the association between duration of concomitant HT and CR after RT. Covariates were selected on the basis of two previously published predictive models that addressed the risk of recurrence after RT [3], [22]. Specifically, covariates consisted of pathologic stage ( $\leq$ pT3a vs $\geq p T 3 b$ ), pathologic Gleason score ( $\leq 7$ vs $\geq 8$ ), and PSA level at RT.

Second, we wished to assess whether CR-free survival differed by duration of concomitant HT by testing an interaction with $\mathrm{HT}$ duration and baseline risk. Three risk factors were identified: pathologic stage $\geq$ pT3b, pathologic Gleason score $\geq 8$, and PSA $>0.5 \mathrm{ng} / \mathrm{ml}$ at RT. Patients were divided into three groups according to their number of risk factors ( 0 vs $1 \mathrm{vs} \geq 2$ ). The choice of interaction variables ( $0 \mathrm{vs}$ $1 \mathrm{vs} \geq 2$ ) was prespecified. We evaluated the number of risk factors to give the reader a clear message easily applicable to clinical practice, instead of evaluating each variable separately.

Third, the Kaplan-Meier method was used to assess CR-free survival for the three groups. The log-rank test was used to test the hypothesis that CR-free survival was similar among groups.

Fourth, locally weighted 8-yr Kaplan-Meier estimates using values from a continuous covariate (stLowess) method were used to explore graphically the relationship between duration of concomitant HT and the CR-free survival rate at $8 \mathrm{yr}$ after RT. The same graph was stratified according to the number of pathologic risk factors ( 0 vs 1 vs $\geq 2$ ).

All statistical analyses were performed using Stata version 12.0 (StataCorp LP, College Station, TX, USA). 


\section{Results}

Overall, 1125 patients (89\%) received SRT for rising PSA, whereas 139 (11\%) were treated for PSA persistence after RP. Descriptive characteristics for the patient population are reported in Table 1. Concomitant HT was administered to 363 patients (29\%), with a median HT duration of 9 mo (IQR 418).

At a median follow-up of 93 mo after surgery, 182 patients developed CR. The CR-free survival rate at 8-yr follow-up was $92 \%$ (95\% confidence interval [CI] 90-94\%). Multivariable Cox regression analysis (Table 2) revealed that concomitant HT duration was inversely associated with the risk of CR (hazard ratio [HR] per 2 mo $0.95,95 \% \mathrm{Cl} 0.92-0.99 ; p=0.022$ ). Moreover, pathologic stage $\geq p$ T3b (HR 3.79; $p<0.0001$ ), pathologic Gleason score $\geq 8$ (HR 1.99; $p<0.0005$ ), and PSA level at RT (HR 1.18; $p=0.015$ ) were also significantly associated with the risk of CR after RT. As outlined, these three variables were considered risk factors; in the data set, 531 patients (42\%) had zero, 507 (40\%) had one, and $226(18 \%)$ two or more risk factors. According to the Kaplan-Meier method (Fig. 1) the 8-yr CR-free survival rate was $94 \%, 89 \%$, and $71 \%$ for men with zero, one, and two or more risk factors, respectively $(p<0.0001)$.

The association between CR-free survival and duration of concomitant HT differed significantly by the number of risk factors ( 0 vs $1, p=0.001 ; 0$ vs $\geq 2, p<0.0001$ on interaction test).

Using the st-Lowess method, we evaluated the relationship between HT duration and the CR rate at $8 \mathrm{yr}$. We observed a significant effect of HT duration for patients with two or more risk factors, for whom HT administration was beneficial when given for up to $36 \mathrm{mo}$. This effect was attenuated for patients with one risk factor, for whom concomitant HT was slightly beneficial when administered for a short time $(<12 \mathrm{mo})$. Conversely, for patients with no risk factors, the risk of CR remained low and constant regardless of HT duration (Fig. 2). The same analyses were repeated for patients with undetectable PSA after surgery $(n=1125 ; 89 \%)$ and excluding those with PSA persistence $(n=139$; $11 \%)$. The results were comparable to the findings obtained for the overall patient population (Supplementary Fig. 1).

We then tested the hypothesis that the impact of HT differed significantly by SRT dose. On multivariable analysis, SRT dose was not significantly associated with the risk of CR (HR 1.10; $p=0.2$ ). Furthermore, the effect of administration of concomitant HT did not significantly differ by SRT dose $(p=0.5$, interaction test; Supplementary Fig. 2).

\section{Discussion}

The hypothesis of the current study was that the impact of HT duration during post-RP RT varies significantly according to the clinical and pathologic characteristics of the patient. Our results confirmed this hypothesis, because the effect of concomitant HT duration on CR risk after RT differed according to clinical and pathologic features. Several facets of these findings deserve particular attention.

First, the novelty of this study was in testing the impact of HT duration by patient characteristics. Two RCTs have shown that addition of ADT to post-RP RT improves oncologic outcomes among men with unfavorable prostate cancer [8], [9]. In particular, the RTOG 9601 study compared RT plus ADT (150 mg of bicalutamide during and after RT for $2 \mathrm{yr}$ ) with RT alone [8]. An improvement in overall survival was observed in the combination group after median follow-up of $12.6 \mathrm{yr}$. However, the greatest survival benefit was seen among patients with more aggressive prostate cancer, such as those with high PSA at trial entry $(1.5-4.0 \mathrm{ng} / \mathrm{ml})$. Similarly, the GETUG-AFU 16 study was a phase 3 RCT that assessed the effect of adding ADT to post-RP RT [9], with short-term (6 mo) administration of a luteinizing hormone releasing hormone agonist (goserelin) as HT. In this trial, patients with more favorable characteristics were selected at the time of randomization: all patients had an undetectable PSA concentration of $<0.1 \mathrm{ng} / \mathrm{ml}$ after RP, and an upper PSA limit of $2 \mathrm{ng} / \mathrm{ml}$ at the time of SRT. At median follow-up of 
$53 \mathrm{mo}$, there was no significant effect of goserelin administration on overall survival. On the contrary, 5 -yr progression-free survival was significantly higher in the combination group, at $80 \%$ compared to $62 \%$. Considering the side-effects of ADT that have been described (cardiovascular morbidity [10], [11], metabolic effects [12], nonmetastatic bone fractures [13], [14], sexual dysfunction [15], hot flushes [16], fatigue, and neurologic side effects [17]), the results from the RTOG 9601 and GETUG-AFU 16 trials suggest a need for appropriate patient selection when concomitant HT is administered. In this setting, we observed a significant interaction between concomitant HT duration and the baseline risk of CR calculated via a multivariable model. The observation that the impact of concomitant HT varies according to its duration and to disease aggressiveness supports the hypothesis that the potential benefit of adding ADT to RT might be related to an effect on subclinical metastatic disease outside the radiation field rather than to a failure of RT to maximize local disease control [8], [9], [23], [24].

Second, our findings have implications for clinical practice. We identified three risk factors ( $p$ T stage $\geq \mathrm{pT} 3 \mathrm{~b}$, pathologic Gleason score $\geq 8$, and PSA $>0.5 \mathrm{ng} / \mathrm{ml}$ at RT) that can be used to identify patients who might benefit the most from HT associated with post-RP RT. In our analyses, long-term ADT was highly beneficial for patients with two or more risk factors. Among these patients, CR risk was approximately halved going from 0 to $12 \mathrm{mo}$, with a further $25 \%$ reduction in relative risk going from 12 to 18 mo for HT duration. Similarly, for patients with one risk factor the CR rate was significantly lower among those treated with short-term HT (ie, $\leq 12 \mathrm{mo}$ ), whereas a clear benefit of long-term HT (ie, $>12 \mathrm{mo}$ ) was not observed. Finally, the absolute risk of CR remained constant for patients without risk factors, regardless of HT duration.

Although subgroup analyses of RCTs should be interpreted with caution, our results are in line with those observed in the RTOG 9601 study, whereby patients with higher PSA levels at SRT appeared to experience the greatest benefit in terms of survival from addition of bicalutamide [8]. Conversely, few retrospective studies have demonstrated a biochemical outcome benefit from addition of ADT to adjuvant or salvage RT [23], [24], [25], [26], [27]. Jackson et al. [23] compared a combination of ADT and RT with RT alone. Several hormonal therapy regimens had been administered in the ADT group, with more than $80 \%$ of patients receiving a gonadotropin-releasing hormone agonist with or without a nonsteroidal antiandrogen. No significant differences were observed between the two groups in terms of distant metastasis and cancer-specific and overall mortality, even though patients treated with combination therapy had more aggressive disease. Further analyses were performed for the ADT group to assess the effect of HT duration on cancer control. A threshold of 12 mo was used to split the combination therapy group into two subgroups. When compared with $<12 \mathrm{mo}$, ADT duration of $>12$ mo was significantly associated with lower biochemical recurrence and CR.

Third, the study included a large $(n=1590)$ multi-institutional series of patients with median follow-up of $93 \mathrm{mo}$. To the best of our knowledge, this represents one of the largest series of patients treated with post-RP RT. Despite the multi-institutional nature of the study, the clinical target volume and the RT dose were similar among centers. These points represent additional strengths of the study.

Finally, we tested the hypothesis that the impact of HT differs significantly by SRT dose. However, administration of concomitant HT did not show a significantly different effect by SRT dose. This is probably related to the fact that $90 \%$ of the patients received a total SRT dose of $<70$ Gy, whereas patients who received higher doses were affected by more advanced and aggressive disease, leading to potential selection bias. Therefore, this hypothesis should be tested in a properly designed study.

Despite several strengths, our study is not devoid of limitations. First, the retrospective nature might have introduced a selection bias whereby indications for adjuvant and/or salvage RT were not standardized in terms of timing, field, and dose. Second, the lack of a specific protocol for the type of drug selected, timing, and duration of $\mathrm{HT}$ administration represents an additional limitation. However, at the time of RT administration in this historical series, there was no evidence to support the superiority of one ADT scheme over the others. 


\section{Conclusions}

In this study we found that the duration of concomitant $\mathrm{HT}$ had a different impact depending on the clinical and pathologic characteristics of the patients. The benefit in reducing the risk of CR was highest for men with at least two of the following features: pT3b/pT4 disease, pathologic Gleason score $\geq 8$, and PSA $>0.5 \mathrm{ng} / \mathrm{ml}$. Among these patients, the longer the HT duration, the lower was the risk of CR. This effect was attenuated in patients with one risk factor, where the use of concomitant HT was beneficial when administered for a short time (ie, $<1 \mathrm{yr}$ ). Conversely, for patients with no risk factors, the risk of $\mathrm{CR}$ remained constantly low regardless of $\mathrm{HT}$ duration. Prospective randomized clinical trials are needed to corroborate our findings.

Author contributions: Nicola Fossati had full access to all the data in the study and takes responsibility for the integrity of the data and the accuracy of the data analysis.

Study concept and design: Fossati.

Acquisition of data: Soligo, Coraggio, Noris Chiorda, Goldner, Battaglia, Bartkowiak, Fonteyne. Analysis and interpretation of data: Fossati, Robesti, Gandaglia, Scarcella, Briganti.

Drafting of the manuscript: All authors.

Critical revision of the manuscript for important intellectual content: Karnes, Boorjian, Bossi, Di Muzio, Cozzarini, Shariat, Joniau, Haustermans, De Meerleer, Ost, Van Poppel, Montorsi, Wiegel, Briganti.

Statistical analysis: Fossati, Robesti, Gandaglia.

Obtaining funding: None.

Administrative, technical, or material support: None.

Supervision: Van Poppel, Wiegel, Montorsi.

Other: None.

Financial disclosures: Nicola Fossati certifies that all conflicts of interest, including specific financial interests and relationships and affiliations relevant to the subject matter or materials discussed in the manuscript (eg, employment/affiliation, grants or funding, consultancies, honoraria, stock ownership or options, expert testimony, royalties, or patents filed, received, or pending), are the following: None.

\section{Funding/Support and role of the sponsor: None.}


Table 1. Descriptive characteristics for 1099 patients treated with salvage RT

\begin{tabular}{|c|c|c|c|}
\hline \multirow[t]{2}{*}{ Variable } & \multicolumn{2}{|c|}{ Concomitant hormonal therapy } & \multirow[t]{2}{*}{$p$ value } \\
\hline & No & Yes & \\
\hline Patients, $n(\%)$ & $729(66)$ & $370(34)$ & \\
\hline Median age at RP, $\mathrm{yr}$ (IQR) & $64(60-68)$ & $65(60-69)$ & 0.14 \\
\hline Median preoperative PSA, ng/ml (IQR) & $8.1(5.8-12.4)$ & $8.5(6.1-14.0)$ & 0.12 \\
\hline \multicolumn{4}{|l|}{ Pathologic stage, $n(\%)$} \\
\hline pT2c & $417(57)$ & $213(58)$ & 0.5 \\
\hline pT3a & $203(28)$ & $94(25)$ & \\
\hline$\geq \mathrm{pT} 3 \mathrm{~b}$ & $109(15)$ & $63(17)$ & \\
\hline \multicolumn{4}{|l|}{ Pathologic Gleason score, $n(\%)$} \\
\hline$\leq 6$ & $222(30)$ & $99(27)$ & 0.0004 \\
\hline 7 & $377(52)$ & $167(45)$ & \\
\hline$\geq 8$ & $130(18)$ & $104(28)$ & \\
\hline \multicolumn{4}{|l|}{ Surgical margin status, $n(\%)$} \\
\hline Negative & $153(21)$ & $89(24)$ & 0.5 \\
\hline Positive & $372(51)$ & $170(46)$ & \\
\hline Unknown & $204(28)$ & $111(30)$ & \\
\hline \multicolumn{4}{|l|}{ Lymph node status, $n(\%)$} \\
\hline pNo & $526(72)$ & $322(87)$ & $<0.0001$ \\
\hline $\mathrm{pNx}$ & $203(28)$ & $48(13)$ & \\
\hline Median time from RP to PSA rising, mo (IQR) & $23(12-43)$ & $15(12-34)$ & 0.001 \\
\hline Median PSA level at salvage RT, $\mathrm{ng} / \mathrm{ml}$ (IQR) & $0.38(0.20-0.66)$ & $0.40(0.18-0.90)$ & 0.069 \\
\hline Median PSA-DT, mo (IQR) & $10(6-17)$ & $7(3-14)$ & 0.0001 \\
\hline \multicolumn{4}{|l|}{ RT field, $n(\%)$} \\
\hline Prostatic fossa & $494(68)$ & $244(66)$ & 0.5 \\
\hline Whole pelvis & $235(32)$ & $126(34)$ & \\
\hline Median RT dose, Gy (IQR) & $66(64-66)$ & $70(66-74)$ & $<0.0001$ \\
\hline Number of risk factors, $n(\%)$ & & & $<0.0001$ \\
\hline 0 & $361(50)$ & $128(35)$ & \\
\hline 1 & $276(38)$ & $169(46)$ & \\
\hline$\geq 2$ & $92(13)$ & $73(20)$ & \\
\hline
\end{tabular}

$\mathrm{DT}=$ doubling time; $\mathrm{IQR}=$ interquartile range; $\mathrm{PSA}=$ prostate-specific antigen; $\mathrm{RP}=$ radical prostatectomy; $\mathrm{RT}=$ radiation therapy.

a Data available for 441 patients. 
Table 2. Multivariable Cox regression analysis to test the association of concomitant hormonal therapy (per $2 \mathrm{mo}$ ) with clinical and biochemical recurrence among 1099 patients treated with salvage radiation therapy for rising prostate-specific antigen after radical prostatectomy

\begin{tabular}{lcc}
\hline Outcome $^{2}$ & HR $(95 \% \mathrm{CI})$ & $p$ value \\
Clinical recurrence & $0.95(0.93-0.98)$ & 0.001 \\
Biochemical recurrence & $0.90(0.87-0.94)$ & $<0.0001$ \\
\hline
\end{tabular}

$\mathrm{Cl}=$ confidence interval; $\mathrm{HR}=$ hazard ratio.

a Data adjusted for pathologic stage ( $\leq$ pT3a vs $\geq$ pT3b), pathologic Gleason score ( $\leq 7$ vs $\geq 8$ ), and prostate-specific antigen level $(\mathrm{ng} / \mathrm{ml})$. 
Fig. 1. Kaplan-Meier plot depicting clinical recurrence (CR)-free survival among 1264 patients treated with salvage radiation therapy, stratified according to the number of risk factors ( 0 vs 1 vs $\geq 2$ ). (0: red line; 1 : blue line; >=2: orange line)

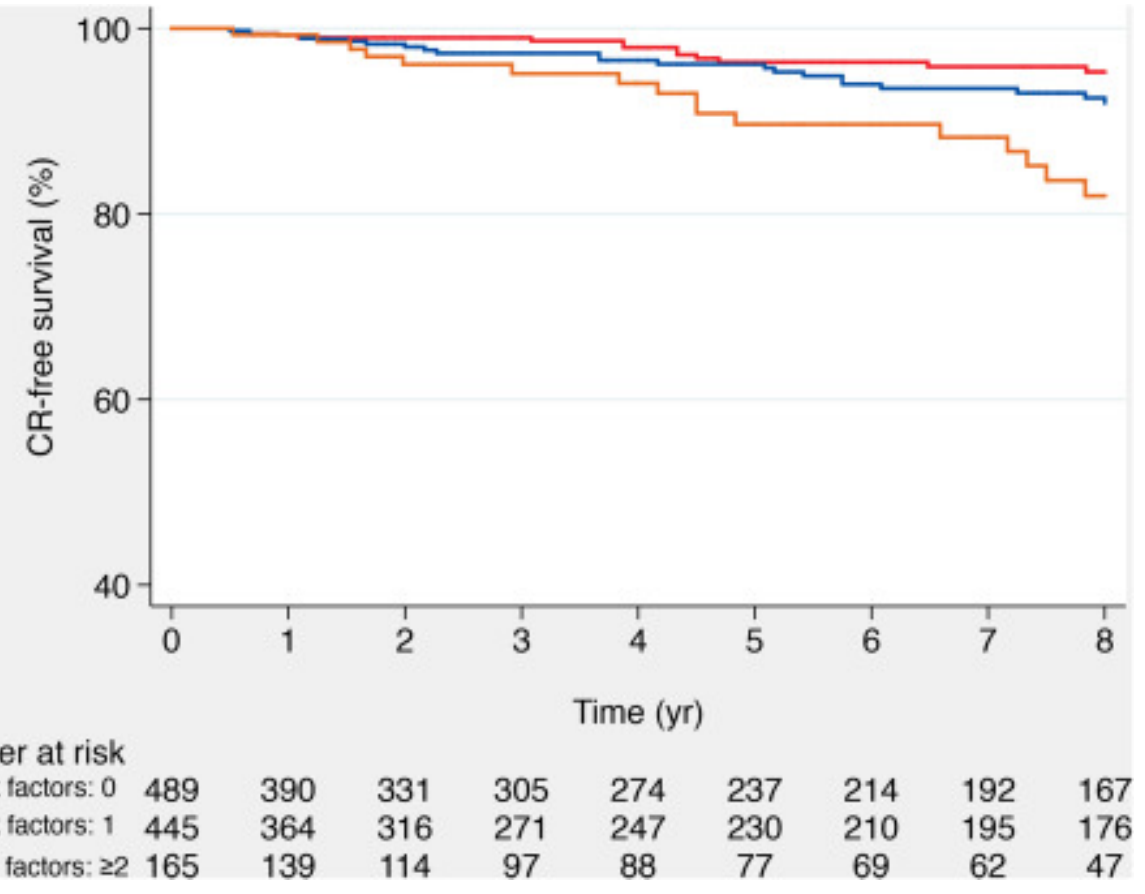

Supplementary Figure 1. Risk of clinical recurrence at $8 \mathrm{yr}$ (derived from multivariable Cox regression analysis) plotted against HT duration. Only patients with undetectable PSA after surgery were included $(n=1125 ; 89 \%$ ). Patients were stratified according to the number of risk factors ( 0 vs. 1 vs. $\geq 2$ )

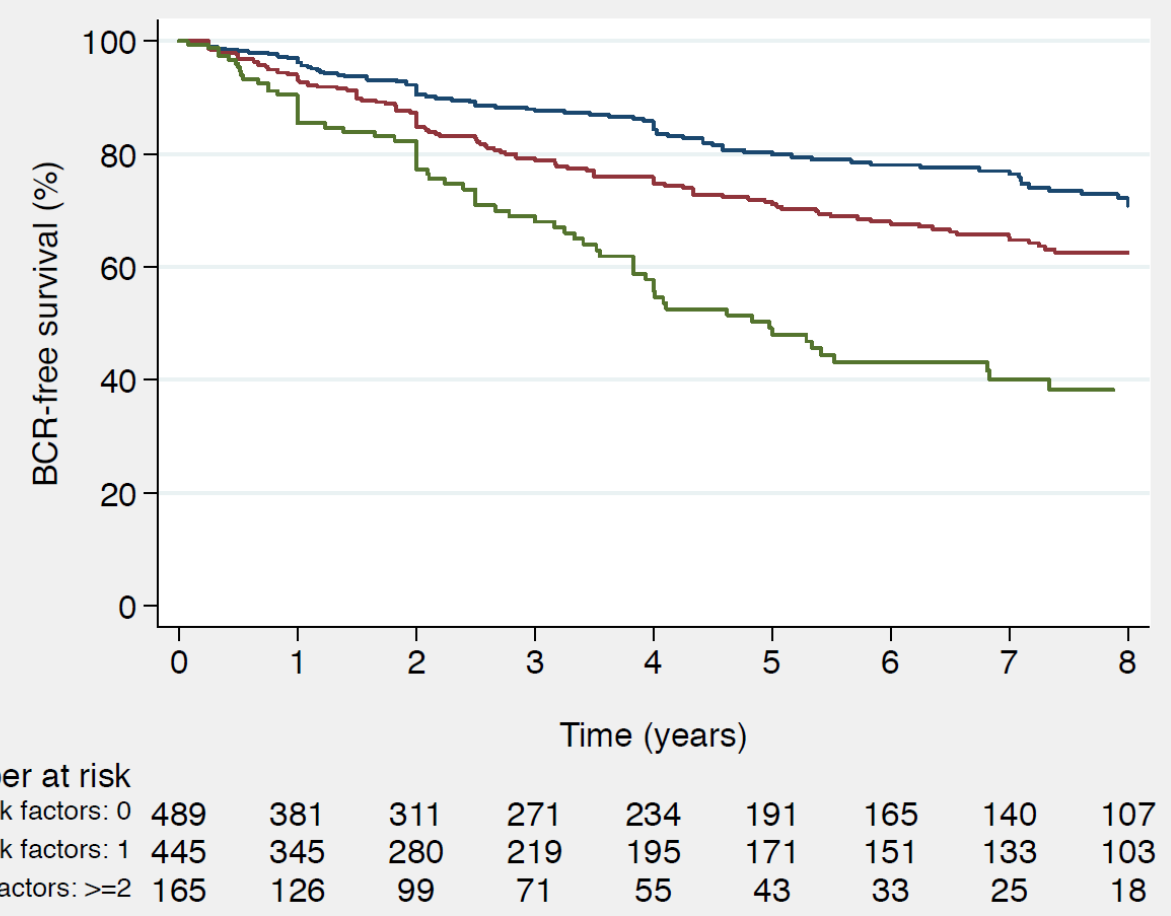


Fig. 2. Risk of clinical recurrence at 8 yr (derived from multivariable Cox regression analysis) plotted against hormone therapy $(\mathrm{HT})$ duration. Patients were stratified according to the number of risk factors (0 vs 1 vs $\geq 2$ ).

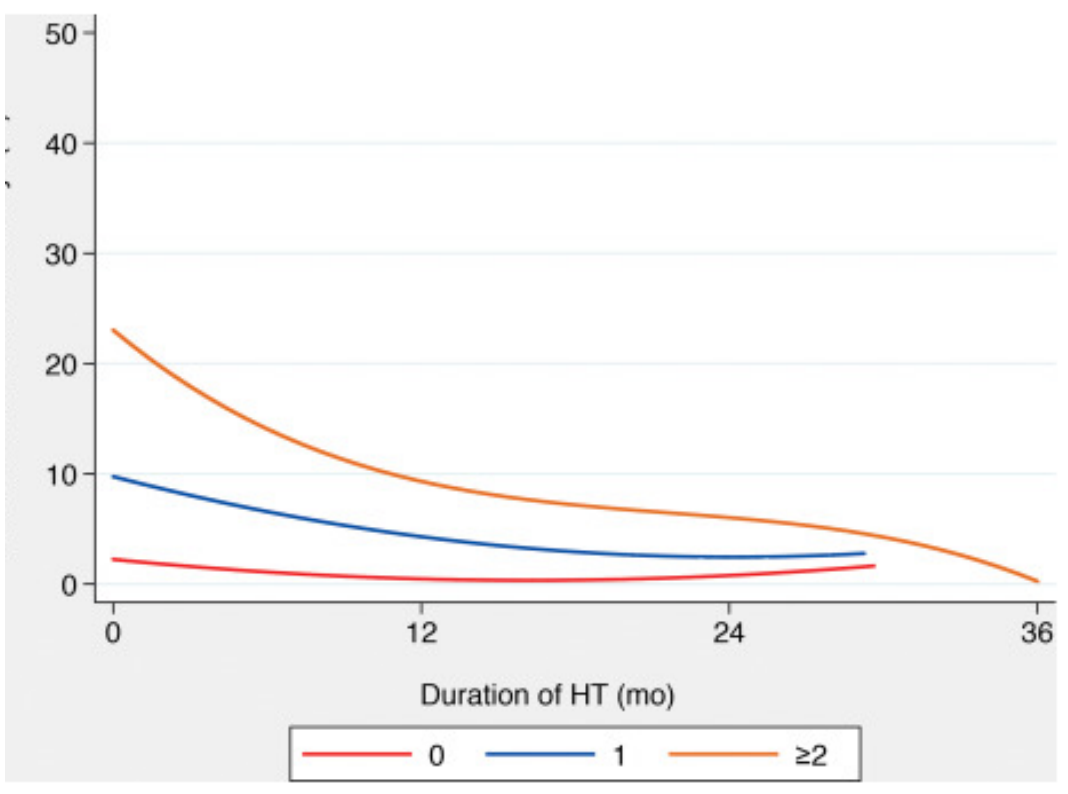

Supplementary Figure 2. Risk of clinical recurrence at 8 yr (derived from multivariable Cox regression analysis) plotted against SRT dose. Patients were stratified according to the use of concomitant HT (no vs. yes).

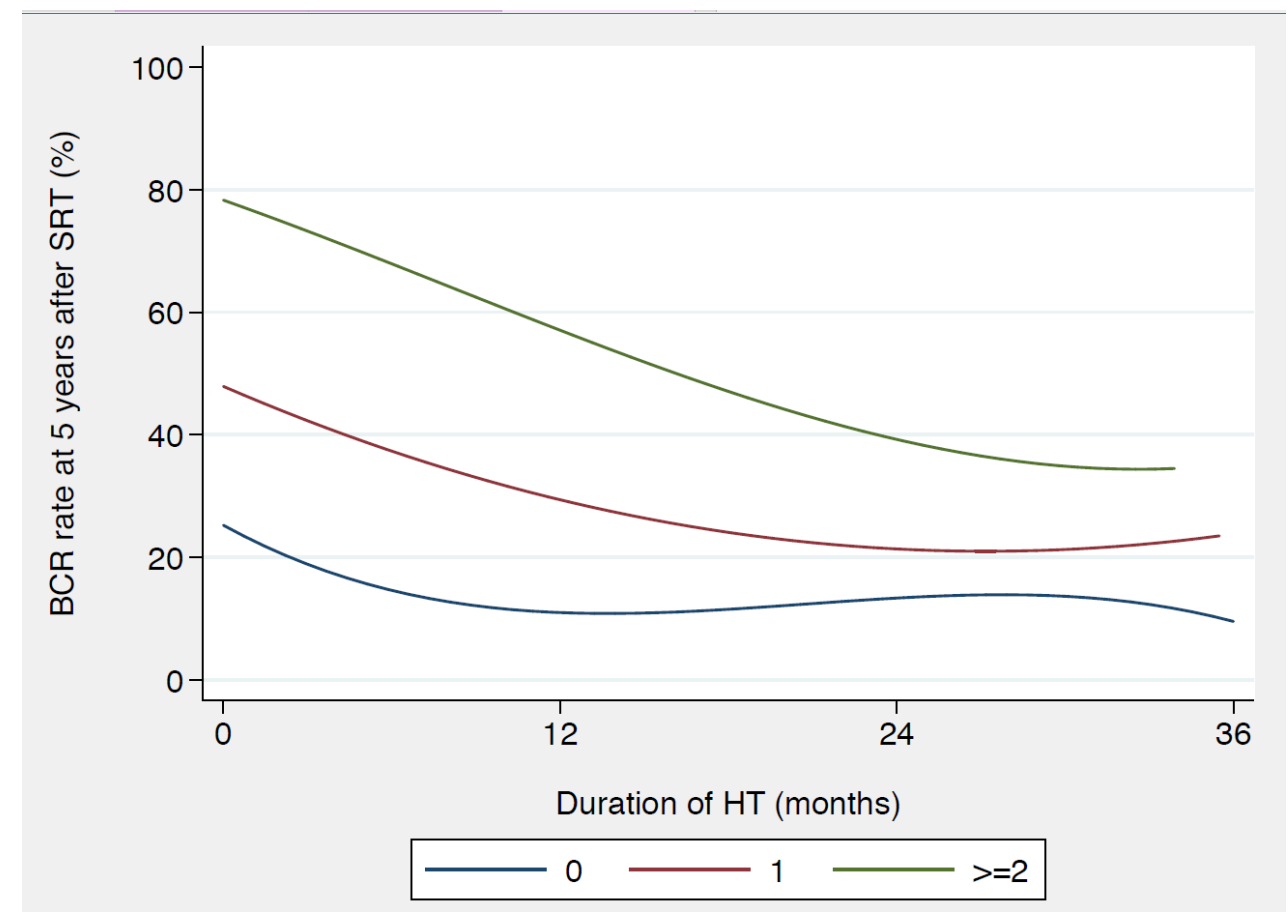




\section{References}

[1]

M.W. Kattan, J.A. Eastham, A.M. Stapleton, T.M. Wheeler, P.T. Scardino. A preoperative nomogram for disease recurrence following radical prostatectomy for prostate cancer.

J Natl Cancer Inst, 90 (1998), pp. 766-771

[2]

A.J. Stephenson, P.T. Scardino, J.A. Eastham, et al. Preoperative nomogram predicting the 10-year probability of prostate cancer recurrence after radical prostatectomy.

J Natl Cancer Inst, 98 (2006), pp. 715-717

[3]

R.D. Tendulkar, S. Agrawal, T. Gao, et al. Contemporary update of a multi-institutional predictive nomogram for salvage radiotherapy after radical prostatectomy.

J Clin Oncol, 34 (2016), pp. 3648-3654

[4]

D. Pfister, M. Bolla, A. Briganti, et al. Early salvage radiotherapy following radical prostatectomy.

Eur Urol, 65 (2014), pp. 1034-1043

[5]

W.C. Jackson, K. Suresh, V. Tumati, et al. Impact of biochemical failure after salvage radiation therapy on prostate cancer-specific mortality: competition between age and time to biochemical failure.

Eur Urol Oncol, 1 (2018), pp. 276-282

[6]

A.U. Kishan, R.D. Tendulkar, P.T. Tran, et al. Optimizing the timing of salvage postprostatectomy radiotherapy and the use of concurrent hormonal therapy for prostate cancer.

Eur Urol Oncol, 1 (2018), pp. 3-18

[7]

D. Bartkowiak, R. Thamm, D. Bottke, et al. Prostate-specific antigen after salvage radiotherapy for postprostatectomy biochemical recurrence predicts long-term outcome including overall survival. Acta Oncol, 57 (2018), pp. 362-367

[8]

W.U. Shipley, W. Seiferheld, H.R. Lukka, et al. Radiation with or without antiandrogen therapy in recurrent prostate cancer.

N Engl J Med, 376 (2017), pp. 417-428

[9]

C. Carrie, A. Hasbini, G. de Laroche, et al. Salvage radiotherapy with or without short-term hormone therapy for rising prostate-specific antigen concentration after radical prostatectomy (GETUG-AFU 16): a randomised, multicentre, open-label phase 3 trial.

Lancet Oncol, 17 (2016), pp. 747-756

[10]

G. Lu-Yao, T.A. Stukel, S.-L. Yao. Changing patterns in competing causes of death in men with prostate cancer: a population based study.

J Urol, 171 (2004), pp. 2285-2290 
[11]

N.L. Keating, A.J. O'Malley, S.J. Freedland, M.R. Smith. Diabetes and cardiovascular disease during androgen deprivation therapy: observational study of veterans with prostate cancer.

J Natl Cancer Inst, 102 (2010), pp. 39-46

[12]

P.J. Saylor, M.R. Smith. Metabolic complications of androgen deprivation therapy for prostate cancer. J Urol, 181 (2009), pp. 1998-2006

[13]

M.R. Smith, S.P. Boyce, E. Moyneur, M.S. Duh, M.K. Raut, J. Brandman. Risk of clinical fractures after gonadotropin-releasing hormone agonist therapy for prostate cancer.

J Urol (2006)

[14]

M. Cree, C.L. Soskolne, E. Belseck, et al. Mortality and institutionalization following hip fracture.

J Am Geriatr Soc, 48 (2000), pp. 283-288

[15]

S. Elliott, D.M. Latini, L.M. Walker, R. Wassersug, J.W. Robinson. Androgen deprivation therapy for prostate cancer: recommendations to improve patient and partner quality of life

J Sex Med, 7 (2010), pp. 2996-3010

[16]

P.L. Nguyen, S.M.H. Alibhai, S. Basaria, et al. Adverse effects of androgen deprivation therapy and strategies to mitigate them.

Eur Urol, 67 (2015), pp. 825-836

[17]

F. Meng, S. Zhu, J. Zhao, et al. Stroke related to androgen deprivation therapy for prostate cancer: a meta-analysis and systematic review.

BMC Cancer, 16 (2016), p. 180

[18]

F.C. Da Silva, F.M.C. Da Silva, F. Gonçalves, et al. Locally advanced and metastatic prostate cancer treated with intermittent androgen monotherapy or maximal androgen blockade: results from a randomised phase 3 study by the South European Uroncological Group.

Eur Urol, 66 (2014), pp. 232-239

[19]

P.C.M.S. Verhagen, M.F. Wildhagen, A.M. Verkerk, et al. Intermittent versus continuous cyproterone acetate in bone metastatic prostate cancer: results of a randomized trial.

World J Urol, 32 (2014), pp. 1287-1294

[20]

J.L. Mohler, A.J. Armstrong, R.R. Bahnson, et al. Prostate cancer, version 1.2016.

J Natl Compr Canc Netw, 14 (2016), pp. 19-30 
[21]

N. Mottet, J. Bellmunt, M. Bolla, et al. EAU-ESTRO-SIOG guidelines on prostate cancer. Part 1: screening, diagnosis, and local treatment with curative intent.

Eur Urol, 71 (2017), pp. 618-629

[22]

A. Briganti, R.J. Karnes, S. Joniau, et al. Prediction of outcome following early salvage radiotherapy among patients with biochemical recurrence after radical prostatectomy.

Eur Urol, 66 (2014), pp. 479-486

[23]

W.C. Jackson, M.J. Schipper, S.B. Johnson, et al. Duration of androgen deprivation therapy influences outcomes for patients receiving radiation therapy following radical prostatectomy.

Eur Urol, 69 (2016), pp. 50-57

[24]

G. Gandaglia, N. Fossati, R.J. Karnes, et al. Use of concomitant androgen deprivation therapy in patients treated with early salvage radiotherapy for biochemical recurrence after radical prostatectomy: long-term results from a large, multi-institutional series.

Eur Urol, 73 (2018), pp. 512-518

[25]

J.W. Jang, W.T. Hwang, T.J. Guzzo, et al. Upfront androgen deprivation therapy with salvage radiation may improve biochemical outcomes in prostate cancer patients with post-prostatectomy rising PSA. Int J Radiat Oncol Biol Phys, 83 (2012), pp. 1493-1499

[26]

D.E. Soto, M.N. Passarelli, S. Daignault, H.M. Sandler. Concurrent androgen deprivation therapy during salvage prostate radiotherapy improves treatment outcomes in high-risk patients.

Int J Radiat Oncol Biol Phys, 82 (2012), pp. 1227-1232

[27]

C.R. King, J.C. Presti, H. Gill, J. Brooks, S.L. Hancock. Radiotherapy after radical prostatectomy: does transient androgen suppression improve outcomes?

Int J Radiat Oncol Biol Phys, 59 (2004), pp. 341-347 